\title{
Binaries at the Bottom of the Main Sequence and below
}

\author{
M. Mayor ${ }^{1}$, S. Udry ${ }^{1}$, J.-L. Halbwachs ${ }^{2}$, F. Arenou ${ }^{3}$ \\ 1 Observatoire de Genève, CH-1290 Sauverny, Switzerland \\ 2 Observatoire de Strasbourg UMR 7550, F-67000 Strasbourg, France \\ ${ }^{3}$ DASGAL, Observatoire de Meudon, F-92195 Meudon, France
}

\begin{abstract}
Long-term radial-velocity surveys of G, K and M dwarfs of the solar neighbourhood are presented. The inferred orbital elements are discussed, focusing on the $(e, \log P)$ diagram, the mass-ratio and secondary mass distributions, and on the binary frequency of the studied samples. The proportion of companions to $\mathrm{M}$ dwarfs is found to be not significantly different from the binary frequency among G- and Kdwarf primaries. The mass function of stellar and planetary companions to solar-type stars strongly suggests different formation and evolution mechanisms for the two populations. Finally, beautiful mass-luminosity relations are obtained from low-mass binaries with high-precision radialvelocity measurements and adaptive optics visual data.
\end{abstract}

\section{Introduction}

Stellar duplicity is a key observational parameter for many astrophysical questions. In particular, large radial-velocity (RV) surveys of spectroscopic binaries provide constraints on possible scenarios of stellar formation and evolution through distributions of orbital elements, correlation between them, or occurrence frequency of multiple systems. Moreover, in combination with visual astrometric observations (speckle, adaptive optics, interferometry) the spectroscopic data provide accurate, direct stellar masses and subsequently allow for the determination of a precise mass function and the mass-luminosity relation.

Since about 20 years, systematic searches have been made to determine the distribution of orbital elements of companions to dwarf stars between F7 and M5. The working horses of these RV searches have been the CORAVEL spectrometers (Baranne et al. 1979) in both hemispheres monitoring at a moderate precision $\left(\sim 250 \mathrm{~ms}^{-1}\right)$ the radial velocities of more than 1000 stars of the solar vicinity. In particular, they have provided an almost complete census of spectroscopic binaries (SB) as part of a volume-limited sample of some 570 F7 to K9 dwarf stars (Duquennoy \& Mayor 1991; Halbwachs et al. 2000a). The M part of the survey is also bringing new results (Udry et al. 2000a) to be compared with what is obtained for the G-K stars. Further progress is provided by on-going high-precision RV surveys with ELODIE and FEROS of M dwarfs of the solar close vicinity (Delfosse et al. 1999a). These surveys will be briefly presented in Sect. 2 and their comparative results discussed in Sect. 3. Binaries detected in systematic long-term searches among the members of open clusters can also 
provide useful additional information for the mass-ratio distribution, improving the available statistics by combining the data accumulated in clusters with the data from the field stars. They are included in the discussion as well.

Section 4 discusses the mass function of substellar companions to solar-type stars as the main indicator of different formation processes between binary stars and extra-solar giant planets.

Concerning precise mass determination, the combination of adaptive optics measurements with ELODIE high-precision velocities has already provided very precise masses (at the few \% level) for about $20 \mathrm{M}$ dwarfs (Delfosse et al. 1999b; Forveille et al. 1999; Segransan et al. 2000), constraining beautifully the massluminosity relation at the bottom of the main sequence (Delfosse et al. 2000). An example is shown in Sect. 5.

\section{Large Radial-Velocity Binary Surveys}

In the early eighties, systematic RV monitorings were initiated with the two CORAVEL spectrographs (Baranne et al. 1979) for different populations of nearby stars in both hemispheres. The two CORAVEL spectrographs were mounted on the 1-m Swiss telescope at Haute-Provence Observatory (France) and on the 1.54-m Danish telescope at La Silla Observatory (Chile). The modest precision of these instruments of about $0.25 \mathrm{kms}^{-1}$ have already permitted a binarity study of close dwarf stars, namely the G, K and M dwarfs of the solar neighbourhood. Originally the stars were taken from the Catalogue of Nearby Stars (CNS2, Gliese 1969) and from its supplement (Gliese \& Jahreiss 1979). They were selected using trigonometric parallaxes derived from on-ground observations, expected to give unbiased volume-limited samples. The results are summarized in following subsections.

The definition of the survey samples is of prime importance for obtaining unbiased estimates of the binary frequency and the distribution of mass ratios. For field stars, a volume-limited sample is a mandatory condition to avoid the well known over-representation in the mass-ratio distribution of binaries with $\Delta m$ close to zero. Precise parallaxes provided by the Hipparcos astrometric mission (ESA 1997) have been of fundamental importance in the last years to more carefully redefine our stellar samples.

\subsection{The CORAVEL G- and K-Dwarf Surveys}

The G-Dwarf Sample. The statistical study of the G-dwarf part of the sample was initially presented in Duquennoy \& Mayor (1991, DM91). Thanks to the rich data collected for the nearby stars (visual and astrometric companions, common proper motion stars) this survey is rather complete, at least for the detection of stellar companions. Part of the 269 stars of that sample, a smaller volume-limited subset of 164 stars, has allowed DM91 to derive:

1) The distribution of periods on a broad domain. The mode of the distribution $f(\log P)$ appears at around 180 years (Fig. 7 of DM91).

2) The distribution of orbital eccentricities as a function of the orbital periods (Fig. 5 of DM91). The main characteristics of the $(e, \log P)$ distribution still hold with additional recent data (see Sect. 3). 
3) The distribution of mass ratios $f(q) \equiv f\left(m_{2} / m_{1}\right)$ for the complete sample (all periods; Fig. 10 of DM91) whose most important characteristics were: a maximum value of the distribution for $q \sim 0.2$ and a declining distribution up to $m_{2} / m_{1}=1$ (no peak close to $q=1$ ). A rediscussion of that material but restricted to $\mathrm{SB}$ with periods $P \leq 3000$ days revealed a flat distribution $(f(q)=$ const; Mazeh et al. 1992). Note that in 1991 the mass distribution for substellar companions was completely unknown.

4) The global percentage of binaries (or multiple stars) in the solar vicinity is close to $2 / 3$.

The Combined $G+K$-Dwarf Sample. The extension of the sample to K dwarfs (Halbwachs et al. 1998), the better definition of the volume-limited sample thanks to the precise Hipparcos parallaxes, the determination of the companion real masses by using Hipparcos astrometric data (Halbwachs et al. 2000b) and the enlargement of the span of our RV survey have been significant steps towards improved orbital-element distributions for companions to solar-type stars. The final analysis of the combined G-K sample is about to be published (Halbwachs et al. in prep). Some aspects are already presented in Halbwachs et al. (2000a) and the distributions of masses and periods will be further discussed in Sect. 3 .

\subsection{M-Dwarf Surveys}

Despite the quoted importance of information on very low-mass star duplicity, systematic spectroscopic studies of the binarity of $M$ dwarfs have been very sparse. Few orbital parameters have been derived so far and the binarity frequency was generally estimated from a statistical treatment of the RV variations (Young et al. 1987; Marcy \& Benitz 1989). The existing studies suffer from small number statistics and large, uncertain, incompleteness corrections. A broad discussion of M dwarfs within $20 \mathrm{pc}$ may also be found in Fischer \& Marcy (1992).

The CORAVEL M-Dwarf Surveys. Preliminary results of a long-term RV search for nearby M-dwarf companions with the 2 CORAVEL, in both hemispheres, are reported in Udry et al. (2000a). The CORAVEL M-dwarf samples consist of about 450 stars of the solar vicinity. Although not complete yet these surveys are already drastically improving the data available for M-dwarf SB by providing a first set of orbital elements for 31 binaries (among which 23 are new).

The High-Precision M-Dwarf Surveys. The situation is also improving with the results of a high-precision RV survey initiated by Delfosse et al. (1998a, 1999a) in 1995 with the ELODIE spectrograph (Baranne et al. 1996) at the HauteProvence Observatory. A systematic RV monitoring of all northern $\mathrm{M}$ dwarfs closer than $\sim 10 \mathrm{pc}$ is in progress to get the first unbiased view of the low-mass star duplicity. For the moment only a first estimate of the occurrence frequency of short-period M-dwarf binaries is available and can be compared with more massive primaries (see Sect. 3). This survey has nevertheless already been very fruitful by revealing previous unknown companions to our closest neighbours (Delfosse et al. 1999a) and a first planet orbiting the low-mass M4 dwarf Gl 876 (Delfosse et al. 1998b; also found by Marcy et al. 1998). Adding new SB orbits from the high-precision survey (Delfosse et al. 1999ab; Forveille et al. 1999) and from the literature to the CORAVEL data, we gather $48 \mathrm{M}$-dwarf orbits 

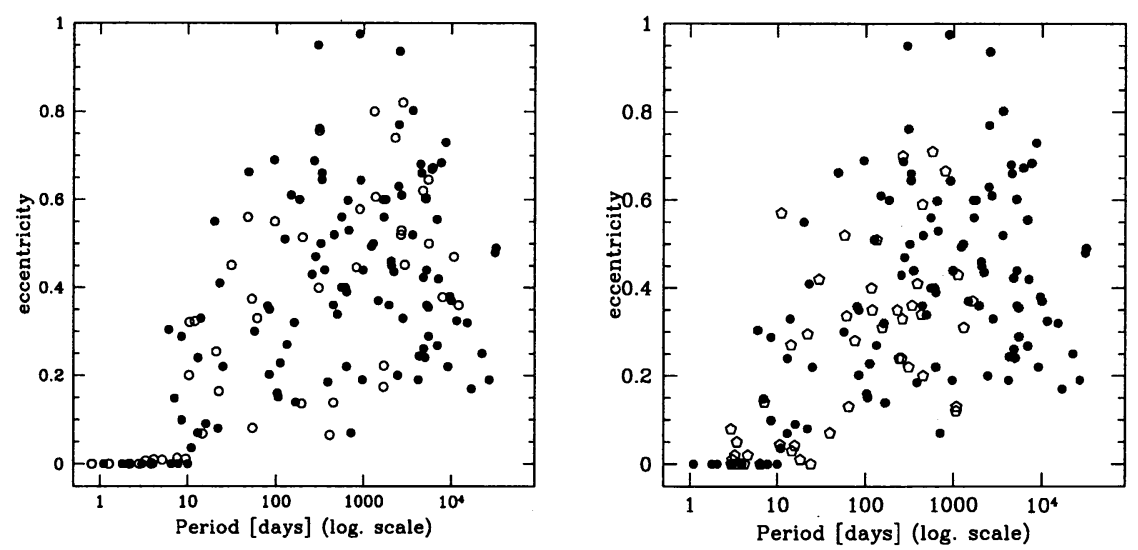

Figure 1. Comparative $(e, \log P)$ diagrams between: Left: the $\mathrm{M}$ dwarfs (open circles) and $\mathrm{G}$ and $\mathrm{K}$ dwarfs (filled circles) of the solar neighbourhood; Right: binaries (filled circles) and planetary systems (open symbols)

that permit a first qualitative comparison of their orbital properties $((e, \log P)$ diagram) with solar-type stars characteristics (see Sect. 3).

A high-precision planet search around $M$ dwarfs in the south has recently been started with FEROS on the 1.5-m ESO telescope at La Silla by the same group, under the leadership of Thierry Forveille. This programme will also provide, as a by-product, useful information on M-dwarf binary characteristics.

We can anticipate further significant progress in the distributions of orbital elements for companions to solar-type stars thanks to other very large programmes. About $3400 \mathrm{G}$ dwarfs are monitored by a Geneva Observatory, CfA and Tel Aviv University collaboration to obtain an unequaled set of orbital parameters for solar-type stars (see Udry et al. 1998 for preliminary results). Another volume-limited survey of $\sim 1650$ late $\mathrm{F}$ to early $\mathrm{M}$ dwarfs is also ongoing at LaSilla with the CORALIE spectrograph on the 1.2-m Euler Swiss telescope, at a 5-7 $\mathrm{ms}^{-1}$ precision level (Udry et al. 2000b). Already now, a few long-term drifts of RV in the programme have revealed SB of long periods and small amplitudes not detected by previous surveys. Systematic adaptive optics searches for distant companions are also promising progress in the definition of the long-period part of the period distribution.

\section{Orbital-Elements Distributions of Companions to Solar-Type Stars}

\subsection{The $(e, \log P)$ Diagram}

The distribution of orbital eccentricities as a function of periods brings information on formation mechanisms of binaries and their dynamical evolution. The comparison of $(e, \log P)$ diagrams of spectroscopic binaries for $\mathrm{G}$ and $\mathrm{K}$ primaries (DM91, Halbwachs et al. 2000a) with the equivalent distribution for $\mathrm{M}$ 
dwarfs (Udry et al. 2000a) is given in Fig. 1 (left). Qualitatively, no fundamental differences are found between the two star populations. The $(e, \log P)$ diagram seems not to depend on the masses of the primary stars. It presents essentially the usual features already pointed out in DM91.

- The short-period SB with $P \leq P_{\text {circ }} \simeq 10 \mathrm{~d}$ are circularized by tidal interactions. For stars with convective envelopes, turbulent viscosity retarding the equilibrium tides is the most efficient mechanism for spin-orbit synchronism and circularization through tidal friction (Zahn 1992). The limit is well marked for $\mathrm{M}$ dwarfs: the highest period with $e \leq 0.05$ is $9.5 \mathrm{~d}$ whereas the smallest period for eccentric orbits is $10.3 \mathrm{~d}$. $P_{\text {circ }}$ is equivalent for $\mathrm{G}$ and $\mathrm{K}$ dwarfs but with a few "eccentric" stars at smaller periods, possibly experiencing partial circularization (Duquennoy, Mayor \& Mermilliod 1992).

- "Longer"-period systems with small eccentricities are missing. Above $P_{\text {circ }}$ imposed by the tidal circularization, all spectroscopic binaries are eccentric. A lower envelope at about $e=0.1$ is observed. Explanations invoke eccentricity pumping mechanism acting at an early phase when stars have disks.

- The eccentricity scatter increases with period attesting to correlation between $e$ and $P$. An upper envelope to the distribution is easily recognized. It probably results from the Roche lobe limitation at the pre-main sequence time.

\subsection{The Mass-Ratio Distribution: $f(q)$}

Systematic RV surveys carried out over the last 20 years have allowed the detection of numerous stellar companions for the stars in the solar vicinity. Distributions of orbital elements for these SB are reasonably well determined, at least down to the substellar regime $\left(m_{2} \sin i \geq 0.075 \mathrm{M}_{\odot}\right)$. However, the mass function of secondaries is much more sensitive to biases of "badly"-defined samples: non volume-limited samples, volume-limited samples incomplete or based on imprecise parallaxes will all be affected by biases. Unfortunately, the price to pay for the strict definition of a volume-limited sample, complete and based on precise parallaxes, is a drastic reduction of the number of useful SB.

Among our 570 stars of the G+K-dwarf sample, 57 SB have been found with periods less than 10 years. This group of SB is called our extended sample and will serve to derive the lower part of $f(q \leq 0.5)$, not affected by the possible excess of binaries with comparable magnitudes. The CNS2 + complement are almost complete for $\pi \geq 65$ mas $\left(\delta \geq-15^{\circ}\right)$ and are almost unbiased for $\pi \geq 46$ mas (Halbwachs et al. 2000a). This latter unbiased sample consists of 249 stars among which only 26 SB have periods smaller than 10 years. That sample has to be used to define the frequency of binary stars with $q>0.5$.

For double-line spectroscopic binaries (SB2), the measured radial-velocity amplitude ratio directly provides the mass ratio: $q=m_{2} / m_{1}=K_{1} / K_{2}$. For single-line binaries (SB1), from the radial velocities, we only have access to

$$
Y \equiv f(m) / m_{1}=\frac{q^{3} \sin ^{3} i}{(1+q)^{2}} .
$$

For limited-size samples the deconvolution of the distribution from the unknown $\sin i$ is hazardous. However, if the periods are not too short, the orbital-plane inclination can be derived from the Hipparcos astrometric data taking into account the precise orbital elements derived from the RV curve (Halbwachs et 

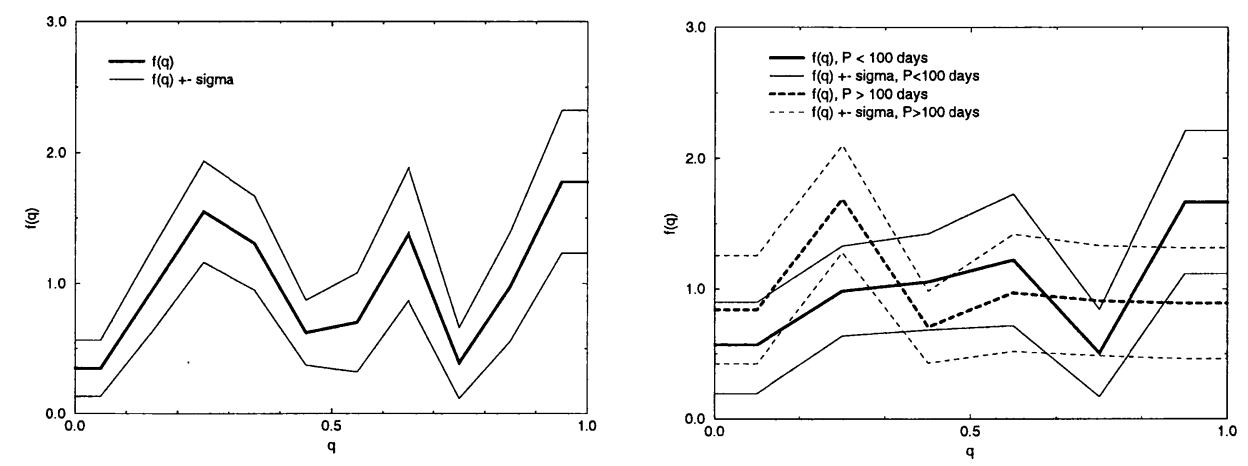

Figure 2. Mass-ratio distributions for: Left: the whole sample (G, K and clusters); Right: 2 domains of periods: $P \leq 100 \mathrm{~d}$ and $P>100 \mathrm{~d}$

al. 2000ab, Arenou et al. 2000). In our 57-SB sample we have 25 SB2. For 22 among the remaining $32 \mathrm{SB} 1$, the astrometric orbit has been solved. Only 10 SB1 are left without mass-ratio direct determination. Finally, an iterative inversion technique is applied (Boffin et al. 1992; Mazeh \& Goldberg 1992).

Star members of open clusters also provide stellar samples convenient to study the mass-ratio distribution. Complete samples of late $\mathrm{F}$ to $\mathrm{G}$ dwarfs members of the Pleiades and Praesepe have been monitored in RV with CORAVEL at the Haute-Provence Observatory during more than 10 years. A set of $11 \mathrm{SB}$ (9 SB1 + 2 SB2) have been found in the Pleiades with periods inferior to 10 years (Mermilliod et al. 1992) and 16 (13 SB1 + 3 SB2) in Praesepe ${ }^{1}$ (Mermilliod \& Mayor 1999). However, for open clusters, photometry is sufficient to set in most cases a fair estimate of the companion masses or at least to set an upper bound (the RV curve gives a lower limit for the secondary mass).

Comparing the binaries in the field and in these clusters, we have a $26 \%$ probability that these two samples are issued from the same parent population. We thus merged the two samples to derive the mass-ratio distribution (Halbwachs et al. 2000a). The global sample is then composed of $84 \mathrm{SB}$ among which only 10 are SB1 without additional constraints. The unbiased part, limited to 46 mas for the field stars and used to analyse the $f(q>0.5)$ side of the distribution, restricts the sample to $50 \mathrm{SB}$ of which only $6 \mathrm{SB} 1$ without constraints.

Figure 2 (left) presents the mass-ratio distribution for the whole sample $(P \leq 10 \mathrm{y})$. We see 3 peaks in the distribution, at $q=0.25,0.6$ and 1 . We also observe the significant decrease of the distribution for $q<0.2$. The peak at $q=0.6$ possibly results from binaries with white-dwarf companions. By estimating the statistical significance of the peak at $q=1$, we only can reject a constant distribution at a $11 \%$ level, a percentage not really significant. If we now separately analyse SB with periods smaller and larger than 100 days (but with $P \leq 10 \mathrm{y}$ ), we see that the possible peak of the mass-ratio distribution at $q=1$, if real, comes from the "shorter-period" binaries ( $P \leq 100$ d; Fig. 2 right).

\footnotetext{
${ }^{1}$ The SB2 percentage is smaller than for the field due to the relative faintness of cluster stars
} 


\subsection{The Period Distribution and Binary Frequency}

From the enlarged $\mathrm{G}+\mathrm{K}$ sample we observe a rise of $f(\log P)$ up to $P \sim 10$ years. The full analysis including visual binaries and common proper-motion stars has still to be finalized to improve on the $f(\log P)$ distribution of DM91.

We have found $26 \mathrm{SB}$ in the unbiased sample $(\pi \leq 46 \mathrm{mas})$ composed of 249 F7-K9 main-sequence primaries. A statistical correction of $0.8 \pm 0.5$ "star" has been estimated for the undetected-binary selection effect. In conclusion, for $q>0.02$ and $P \leq 10$ years, we observe $11 \pm 2 \%$ of double stars among G-K stars in the field. The percentage is $9 \pm 2 \%$ for $P \leq 1000$ days.

For comparison, the percentage of SB detected among close $M$ dwarfs is $8 \pm 3 \%$ for $P \leq 1000$ days (Delfosse \& Forveille 2000). We thus do not observe a significant smaller percentage of SB among $M$ stars compared to the solar-type stars, at least for the unbiased domain of periods $(P \leq 1000 \mathrm{~d})$.

\section{The Mass Function below the Main Sequence}

The moderate precision programmes have already revealed a few companions with $m_{2} \sin i$ small enough to be brown-dwarf candidates (Latham et al. 1989; DM91; Mayor et al. 1997). At the present time, the spectrographs with higherprecision capabilities have brought much further insight into orbital-element distributions and mass function of secondaries with minimum masses below $0.1 \mathrm{M}_{\odot}$. In particular, more than 50 companions of stars of the solar vicinity have been detected with $m_{2} \sin i$ smaller than $20 \mathrm{M}_{\mathrm{Jup}}$, most of them having minimum masses lower than $5 \mathrm{M}_{\mathrm{Jup}}$. The information collected so far on $\mathrm{SB}$, combined with the most recent detection of very low-mass companions (planets and browndwarf candidates), gives us a synthetic view on companions to solar-type stars from $q=m_{2} / m_{1}=1$ to $q<0.001$ (Fig. 3, right).

The histogram of minimum masses for solar-type star companions with $m_{2} \sin i$ less than $0.1 \mathrm{M}_{\odot}$ is drawn in Fig. 3 (left). The impressive peak for $m_{2} \sin i \leq 8 \mathrm{M}_{\text {Jup }}$ is certainly not part of the very flat tail of the stellar and browndwarf secondaries. Despite the huge observational bias against the detection of small mass companions we observe an increasing number of low-mass gaseous planets. As the same time, brown-dwarf candidates (easier to detect) are rare.

In addition to RV data, we have collected information constraining the orbital-plane inclinations of brown-dwarf and planetary candidates (see Mayor \& Udry 2000 for a review): Hipparcos astrometry (Halbwachs et al. 2000a, Zucker \& Mazeh 2000), planetary transit (Charbonneau et al. 2000, Henry et al. 2000, Mazeh et al. 2000), dust disks around stars (Trilling, Brown \& Rivkin 2000) or synchronization properties (Fuhrmann, Pfeiffer \& Bernkopf 1998). For these companions we obtain the real masses, most of them finally in the stellar domain at the bottom of the main sequence. The latter possibly represent the tail of the binary $\sin i$ distribution of the peak at $f(q=0.2)$. Only 4 objects with $\sin i$ determination have real-mass estimates in the brown-dwarf domain (3 from Halbwachs et al. 2000b and 1 from Zucker \& Mazeh 2000).

Taking into account available orbital inclinations a composite histogram is built up for masses $m_{2}$ or $m_{2} \sin i$ (if $\sin i$ not known) (Figs. 3 bottom). The gap between SB and planets is remarkable, emphasizing the paucity of brown-dwarf 

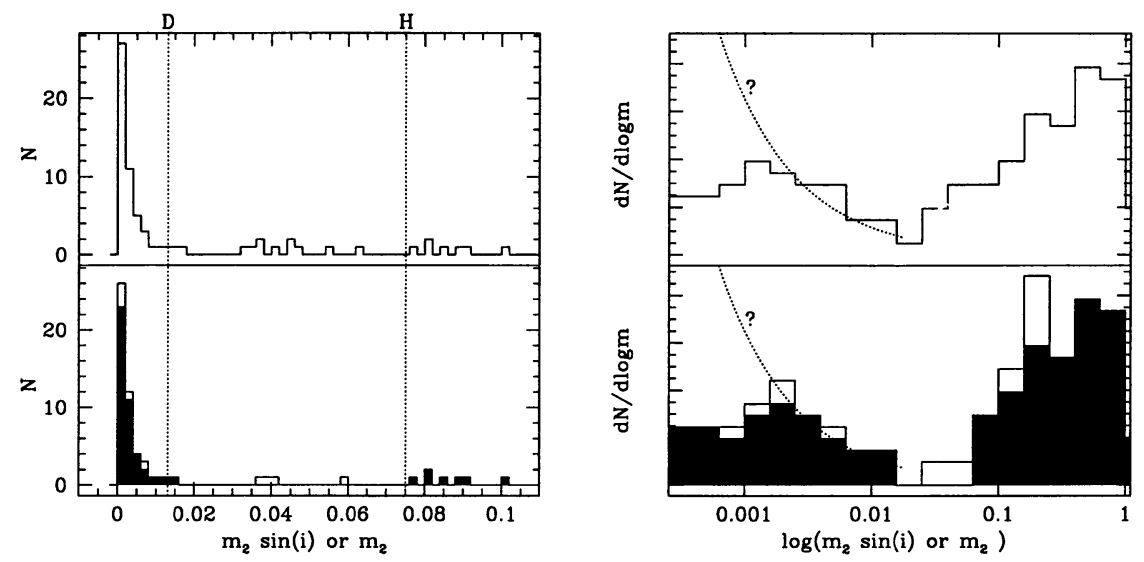

Figure 3. Mass function of companions to solar-type stars. Left: Linear scale. The dotted lines indicate the $\mathrm{H}-\left(75 \mathrm{M}_{\text {Jup }}\right)$ and the D$\left(13 \mathrm{M}_{\mathrm{Jup}}\right)$ burning limits. Right: Log scale. The dotted line is indicative of a potential bias-corrected distribution. For both diagrams: Top: $m_{2} \sin i$. Bottom: composite histogram of $m_{2}$ (open part) and $m_{2} \sin i$ (if $\sin i$ not known; colored part)

companions. It clearly suggests different formation and evolution processes for the two populations. Further indications for such differences have been pointed out in the period and eccentricity distributions (Mayor \& Udry 2000).

The sharp drop of the mass function observed below $\sim 8 \mathrm{M}_{\mathrm{Jup}}$ strongly suggests a maximum mass for giant planets close to $10 \mathrm{M}_{\mathrm{Jup}}$, without any relation with the D-burning limit around $13 \mathrm{M}_{\mathrm{Jup}}$ (Fig. 3 left). The latter has very probably nothing to do with the maximum mass of objects initially formed from dust/ice particles in an accretion disk. At the opposite, present samples cannot provide any real estimate of the minimum mass for brown dwarfs orbiting solartype stars. The D-burning limit seems neither related to the minimum mass of brown dwarfs formed by fragmentation in a cloud. Free-floating "planets" (i.e. brown dwarfs) with masses probably below $10 \mathrm{M}_{\text {Jup }}$ have been reported in the $\sigma$-Orionis cluster (Zapatero Osorio et al. 2000). The D-burning mass is clearly of no help to understand the observed mass distribution.

\section{The Mass-Luminosity Relation at the Bottom of the Main Se- quence}

A superb result of combined studies of low-mass $M$ dwarfs (spectroscopy, adaptive optics imaging) is the determination of the mass-luminosity relation at the bottom of the main sequence. Combined orbits derived from precise RV obtained with ELODIE at a $\sim 10 \mathrm{~ms}^{-1}$ precision level and adaptive optics measurements obtained with PUE'O on the CFHT (Hawaii) and with ADONIS on the 3.6m ESO telescope (LaSilla, Chile) are currently providing masses of exquisite precision (down below 1\%, Segransan et al. 2000). The derived K-band mass- 


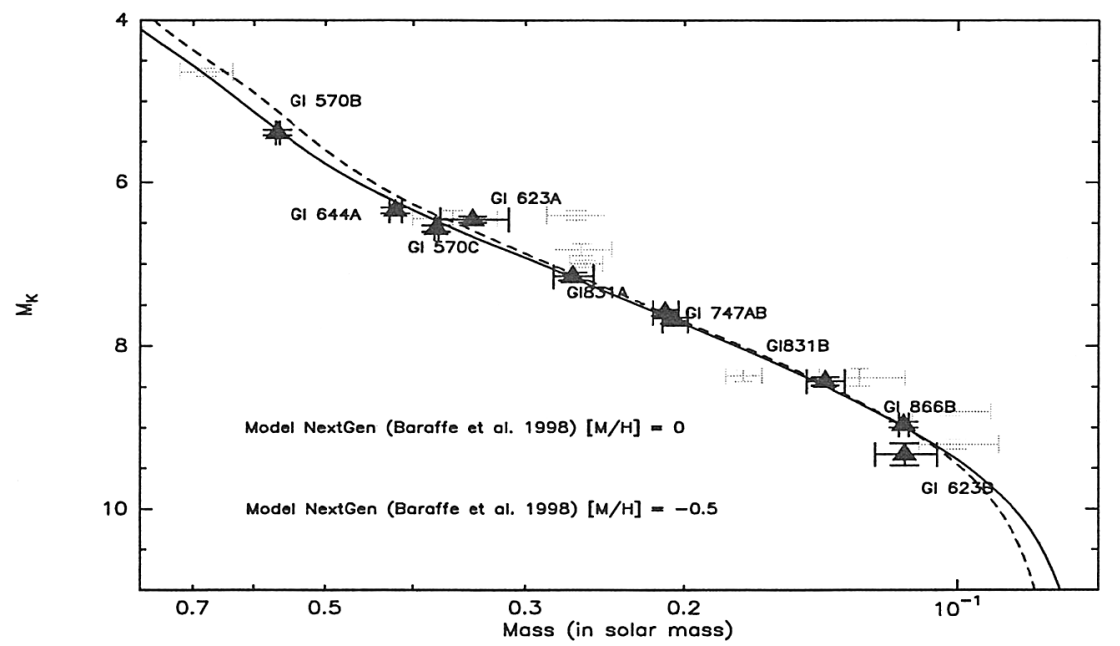

Figure 4. Mass-Luminosity relation in the $\mathrm{K}$ band at the bottom of the main sequence taken from Delfosse et al. (2000)

luminosity relation obtained by Delfosse et al. (2000) of unprecedented quality is shown in Fig. 4. In the $\mathrm{V}$ band, a larger scatter is observed. It is very probably due to metallicity effects, much less influential in the $\mathrm{K}$ band where the agreement with the models by Baraffe et al. (1998) is striking.

\section{References}

Arenou, F., Halbwachs, J.-L., Udry, S., Mayor, M. 2000, in Birth and Evolution of Binary Stars, Poster Proc. IAU Symp. 200, ed. B. Reipurth \& H. Zinnecker, 135

Baranne, A., Mayor, M., Poncet, J.-L. 1979, Vistas in Astron., 23, 279

Baranne, A., Queloz, D., Mayor, M., et al. 1996, A\&AS, 119, 373

Baraffe, I., Chabrier, G., Allard, F., Hauschildt, P. H. 1998, A\&A, 337, 403

Boffin, H., Paulus, G, Cerf, N. 1992, in Binaries as Tracers of Stellar Formation, ed. A. Duquennoy \& M. Mayor, Cambridge Univ. Press, 26

Charbonneau, D., Brown, T., Latham, D., Mayor, M. 2000, ApJ, 529, L45

Delfosse, X., Forveille, T. 2000, in Very-low mass stars and brown dwarfs in stellar clusters and associations, ed. R. Rebolo, M. R. Zapatero Osorio \& V. Béjar, Cambridge Univ. Press

Delfosse, X., Forveille, T., Perrier, C., Mayor, M. 1998a, A\&A, 331, 581

Delfosse, X., Forveille, T., Mayor, M., et al. 1998b, A\&A, 338, L67

Delfosse, X., Forveille, T., Beuzit, J.-L., et al. 1999a, A\&A, 344, 897

Delfosse, X., Forveille, T., Udry, S., et al. 1999b, A\&A, 350, L39

Delfosse, X., Forveille, T., Segransan, D., et al. 2000, A\&A submitted

Duquennoy, A., Mayor, M. 1991, A\&A, 248, 485 
Duquennoy, A., Mayor, M., Mermilliod, J.-C. 1992, in Binaries as Tracers of Stellar Formation, ed. A. Duquennoy \& M. Mayor, Cambridge Univ. Press, 52

ESA 1997, The Hipparcos and Tycho Catalogue, ESA-SP 1200

Fischer, D., Marcy, G. 1992, ApJ, 396, 178

Forveille, T., Beuzit, J.-L., Delfosse, X., et al. 1999, A\&A, 351, 619

Fuhrmann, K., Pfeiffer, M. J., Bernkopf, J. 1998, A\&A 336, 942

Gliese, W. 1969, Ver. Astron. Rechen. Inst. Heidelberg, 22

Gliese, W., Jahreiss, H. 1979, A\&AS, 38, 423

Halbwachs, J.-L., Mayor, M., Udry, S. 1998, in Brown Dwarfs and Extrasolar Planets, ed. R. Rebolo, E. Martin \& M. R. Zapatero Osorio, ASP Conf. Ser. 134, 308

Halbwachs, J.-L., Arenou, F., Mayor, M., Udry, S. 2000a, in Birth and Evolution of Binary Stars, Poster Proc. IAU Symp. 200, ed. B. Reipurth \& H. Zinnecker., 132

Halbwachs, J.-L., Arenou, F., Mayor, M., Udry, S., Queloz, D. 2000b, A\&A, 355,581

Henry, G., Marcy, G., Butler, P., Vogt, S. 2000, ApJ, 529, L41

Latham, D., Mazeh, T., Stefanik, R., Mayor, M., Burki, G. 1989, Nature, 339, 38

Marcy, G. W., Benitz, K.J. 1989, ApJ, 344, 441

Marcy, G., Butler, P., Vogt, S., Fischer, D., Lissauer, J. 1998, ApJ, 505, L147

Mayor, M., Udry, S. 2000, in Disks, Planetesimals and Planets, ed. F. Garzón, C. Eiroa, D. de Winter \& T. Mahoney, ASP Conf. Ser., in press

Mayor, M., Queloz, D., Udry, S., Halbwachs, J.-L. 1997, in Astronomical and Biochemical Origins of Life in the Universe, ed. C. Cosmovici, S. Browyer \& D. Werthimer, IAU Coll. 161, Editrice Compositori, 313

Mazeh, T., Goldberg, D. 1992, ApJ, 394, 592

Mazeh, T., Goldberg, D., Duquennoy, A., Mayor, M. 1992, ApJ, 401, 265

Mazeh, T., Naef, D., Torres, G., et al. 2000, ApJ, 532, L55

Mermilliod, J.-C., Rosvick, J., Duquennoy, A., Mayor, M. 1992, A\&A, 265, 513

Mermilliod, J.-C., Mayor, M. 1999, A\&A, 352, 479

Segransan, D., Delfosse, X., Forveille, T., et al. 2000, A\&A submitted

Trilling, D. E., Brown, R. H., Rivkin, A. S. 2000, ApJ, 529, 499

Udry S., Mayor M., Latham D., et al. 1998, in Cool Stars, Stellar Systems and the Sun, ed. R. Donahue \& J. Bookbinder, ASP Conf. Ser., 154

Udry, S., Mayor, M., Delfosse, X., et al. 2000a, in Birth and Evolution of Binary Stars, Poster Proc. IAU Symp. 200, ed. B. Reipurth \& H. Zinnecker, 158

Udry, S., Mayor, M., Naef, D., et al. 2000b, A\&A, 356, 590

Young, A., Sadjadi, S., Harlan, E. 1987, ApJ, 314, 272

Zahn, J.-P. 1992, in Binaries as Tracers of Stellar Formation, ed. A. Duquennoy \& M. Mayor, Cambridge University Press, 253

Zapatero Osorio, M.-R., Béjar, V., Martin, E., et al. 2000, Science, in press

Zucker, S., Mazeh, T. 2000, ApJ, 531, L67 